\title{
Operational Readiness of a P.S. Redundant System with Three Types of Failure and Waiting Concept
}

\author{
${ }^{1}$ Sanjay Goel, ${ }^{2}$ Anand Tyagi \\ and ${ }^{3}$ Sachin Kumar \\ ${ }^{1}$ Department of Mathematics, BRCM, Haryana, India \\ ${ }^{2}$ Department of Mathematics, RKGEC, Ghaziabad, U. P., India \\ ${ }^{3}$ Department of Mathematics, K.I.E.T., Ghaziabad, U.P., India
}

\begin{abstract}
This paper deals with a multi-component System (Parallel and Series) which has two Subsystem $T_{1}$ and $T_{2}$ connected in series. Subsystem $T_{1}$ is made up two identical units in Parallel, while $T_{2}$ is made up n-identical units in series. Subsystem $T_{1}$ fails when both of its units fails and $T_{2}$ fails when any one of its unit fail. System goes to complete break down if either both $\mathrm{T}_{1}$ and $\mathrm{T}_{2}$ and any one of $T_{1}$ and $T_{2}$ fails. While on failure of 1-T $T_{1-}$ : one out of two, it goes to reduced efficiency state. Whole system may fail completely from any operable state due to networking failure also. There are three type of failure partial failure (minor), catastrophic (major) and networking failure. All type of failure are assumed to be exponential while repair are distributed generally accept Networking failure which is taken constant. In this paper Author's used S.V technique and L.T of various state probabilities have been evaluated. At last expressions for Cost function, Reliability and Variance of time to failure are obtained by inversion process. Conclusion and result of this paper and few graphical illustrations are also given in the end so as to explain practical utility of the problem.
\end{abstract}

Keywords: Variances of time to failure, exponential and general distribution, mean time to system failure. 


\section{System Configuration}

$\mathrm{T}_{1}$

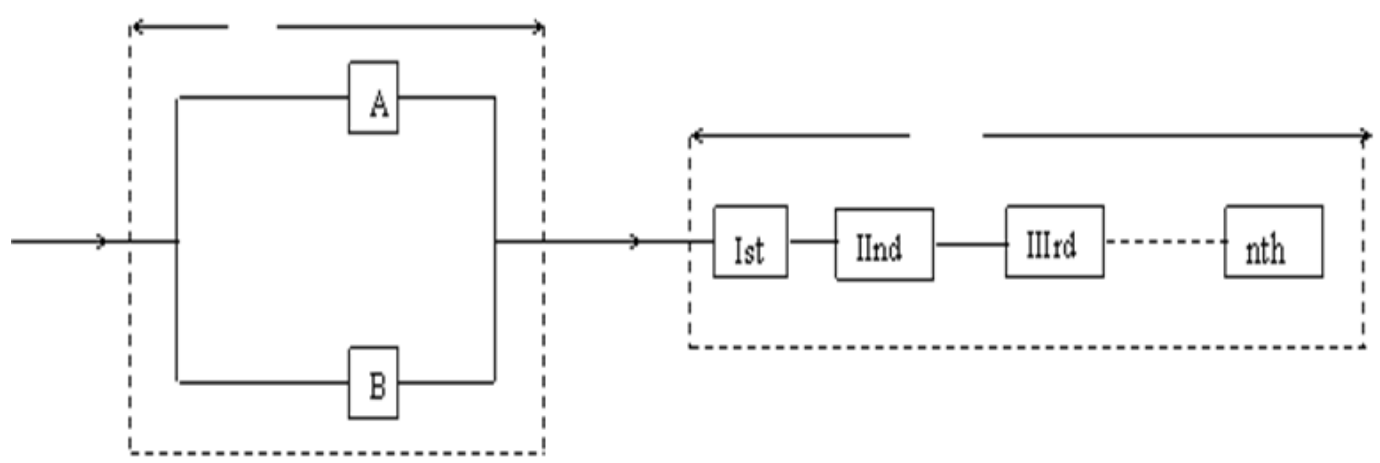

\section{Introduction}

An unreliable system has higher probability of failure and when any item of the associated equipment is damaged or destroyed due to some or other causes, consequently the unreliability of the system may results in to the wastage of cost, time of personal as well as national security. Thus a high degree of reliability is required in various practical system i.e. many equipment of DRDO, Nuclear system, Power plants, Fuel system , Self operating system, Space system. Introducing parallel redundancy and using waiting service facility, is found very useful to achieve the requirement of high degree of reliability’ In 1979 B.S. Dhillon [2];1985 Murty A.S.R, Verma A.K [3] and 1995 Pandey D, Jacob Mendes [4],et all try various reliability technique to achieve a high degree of reliability for system under consideration. But this does not seem to be sufficient and more is required in this direction.

In view of the above authors have tried to fill the gap by considering a higher reliable \& more complex system P.S. redundant system with three types of failure and waiting concept. Mathematical modeling of this model solve by S.V technique and L.T process.

\section{Notations}

$$
\lambda_{m} / \lambda_{m}^{\prime}
$$$$
\lambda_{M}
$$

A

$$
\Phi_{1}(x) / \Phi_{2}(y)
$$

$\mathrm{P}_{0}(t)$

$\mathrm{P}_{1}(x, t) / \mathrm{P}_{2}(t)$
Constant failure rate of any one unit of $T_{1}$ in normal efficiency state / failure rate of $T_{1}$ degraded state.

Constant failure rate of any one unit of subsystem $\mathrm{T}_{2}$

Constant waiting rate for series from $S_{2} \rightarrow S_{3}, S_{4} \rightarrow S_{5}, S_{6} \rightarrow S_{7}$

General repair rates of single unit / two unit at a time with elapsed repair time $\mathrm{x}, \mathrm{y}$ in failed state

The probability that, the system is in operable state at time t.

The probability at time $t$, that the system is under repair in 
degraded states / failed states due to failure of one- unit of $\mathrm{T}_{1} /$ both units of $T_{1}$ and the elapsed repair time lies in the interval $(x, x+\Delta)$ /failure of both- $\mathrm{T}_{1}$ and waiting for expire at the $\mathrm{t}$.

$\mathrm{P}_{3}(y, t) / \mathrm{P}_{5}(y, t) / \mathrm{P}_{7}(x, t) \quad$ The probability at time $\mathrm{t}$ when the system is under repair from state and the elapsed repair time lies in the interval $(y, y+\Delta)$, or $(x, x+\Delta)$

$\mathrm{P}_{4}(t) / \mathrm{P}_{6}(t) \quad$ The probability at time that the system is in failed state due to failure of any $\mathrm{T}_{2}$ - unit.

$\mathrm{P}_{8}(t) \quad$ The probability at time $\mathrm{t}$ when the system is in failed state due to networking failure.

$\delta \quad$ Constant networking failure rate

$\mu \quad$ Immediate constant repair rate for networking failure

$S_{i}(x)=i(x) \cdot e^{-\int_{0}^{x}(x) d x}$ (By Davis formula)

$F_{i}(x)=\frac{1-\bar{S}_{i}(x)}{x}$

\section{Mathematical formulation of this model}

By probability and continuity argument the difference- differential equations for stochastic process which is continuous in time discrete in space are as

$$
\begin{aligned}
& {\left[\frac{d}{d t}+2 \lambda_{m}+n \lambda_{M}+\delta\right] \mathrm{P}_{0}(t)=\int_{0}^{\infty} \mathrm{P}_{1}(x, t) \Phi_{1}(x) d x+\int_{0}^{\infty} \mathrm{P}_{3}(y, t) \Phi_{2}(y) d y} \\
& +\int_{0}^{\infty} \mathrm{P}_{5}(y, t) \Phi_{2}(y) d y+\int_{0}^{\infty} \mathrm{P}_{7}(x, t) \Phi_{1}(x) d x+\mu \cdot \mathrm{P}_{8}(t) \\
& {\left[\frac{\partial}{\partial x}+\frac{\partial}{\partial t}+\lambda_{m}^{\prime}+n \lambda_{M}+\Phi(x)+\delta\right] \mathrm{P}_{1}(x, t)=0} \\
& {\left[\frac{d}{d t}+a\right] \mathrm{P}_{2}(t)=\lambda_{m}^{\prime} \cdot \mathrm{P}_{1}(t)} \\
& {\left[\frac{\partial}{\partial y}+\frac{\partial}{\partial t}+\Phi_{2}(y)\right] \mathrm{P}_{3}(y, t)=0} \\
& {\left[\frac{d}{d t}+a\right] \mathrm{P}_{4}(t)=n \lambda_{M} \mathrm{P}_{1}(t)} \\
& {\left[\frac{\partial}{\partial t}+\frac{\partial}{\partial t}+\Phi_{2}(y)\right] \mathrm{P}_{5}(y, t)=0}
\end{aligned}
$$




$$
\begin{aligned}
& {\left[\frac{d}{d t}+a\right] \mathrm{P}_{6}(t)=n \lambda_{M} \mathrm{P}_{0}(t)} \\
& {\left[\frac{\partial}{\partial x}+\frac{\partial}{\partial t}+\Phi_{1}(x)\right] \mathrm{P}_{7}(x, t)=0} \\
& {\left[\frac{d}{d t}+\mu\right] \mathrm{P}_{8}(t)=\delta\left\{\mathrm{P}_{0}(t)+\int_{0}^{\infty} \mathrm{P}_{1}(x, t) d x\right\}}
\end{aligned}
$$

\section{Boundary Condition}

$$
\begin{aligned}
& \mathrm{P}_{1}(0, t)=2 \lambda_{m} \mathrm{P}_{0}(t) \\
& \mathrm{P}_{3}(0, t)=a \mathrm{P}_{2}(t) \\
& \mathrm{P}_{5}(0, t)=a \mathrm{P}_{4}(t) \\
& \mathrm{P}_{7}(0, t)=a \cdot \mathrm{P}_{6}(t)
\end{aligned}
$$

\section{Initial conditions}

$$
\mathrm{P}_{k}(0)=\left\{\begin{array}{l}
1 \text { if } \mathrm{K}=0 \\
0 \text { if } \mathrm{K}=1,------8
\end{array}\right\} \ldots
$$

\section{Solution of the model}

Taking Laplace transform of equation (1) to (13) and using (14) we get

$$
\overline{\mathrm{P}}_{0}(s)=\frac{1}{A(s)}
$$

where

$$
\begin{aligned}
& A(s)=s+2 \lambda_{m}+n \lambda_{M}+\delta-2 \lambda_{m} \cdot \overline{S_{\Phi_{1}}}\left(s+\lambda_{m}^{\prime}+n \lambda_{M}+\delta\right) \\
& -\frac{2 a \lambda_{m}^{\prime} \lambda_{m}}{(s+a)} F_{\Phi_{1}}\left(s+\lambda_{m}^{\prime}+n \lambda_{M}+\delta\right) \bar{S}_{\Phi_{2}}-2 a \cdot n \lambda_{M} \cdot \lambda_{m} F_{\Phi_{1}}\left(s+\lambda_{m}^{\prime}+n \lambda_{M}+\delta\right) \bar{S}_{\Phi_{2}}(s) \\
& -\frac{n \lambda_{M} \cdot a}{(s+a)} \bar{S}_{\Phi_{1}}(s)-\frac{\delta}{(s+\mu)}\left[1-2 \lambda_{m} \cdot F_{\Phi_{1}}\left(s+\lambda_{m}^{\prime}+n \lambda_{M}+\delta\right)\right] \\
& \overline{\mathrm{P}}_{1}(s)=\frac{2 \lambda_{m}}{\mathrm{~A}(s)} F_{\Phi_{1}}\left(s+\lambda_{m}^{\prime}+n \lambda_{M}+\delta\right) \\
& \overline{\mathrm{P}}_{2}(s)=\frac{2 \lambda_{m}^{\prime} \lambda_{m}}{\mathrm{~A}(s)(s+a)} F_{\Phi_{1}}\left(s+\lambda_{m}^{\prime}+n \lambda_{M}+\delta\right) \\
& \overline{\mathrm{P}}_{3}(s)=\frac{2 a \lambda_{m}^{\prime} \lambda_{m}}{\mathrm{~A}(s)(s+a)} F_{\Phi_{1}}\left(s+\lambda_{m}^{\prime}+n \lambda_{M}+\delta\right)
\end{aligned}
$$




$$
\begin{aligned}
& \overline{\mathrm{P}}_{4}(s)=\frac{2 \lambda_{m} \cdot n \lambda_{M}}{\mathrm{~A}(s)(s+a)} F_{\Phi_{1}}\left(s+\lambda_{m}^{\prime}+n \lambda_{M}+\delta\right) \\
& \overline{\mathrm{P}}_{5}(s)=\frac{2 a \lambda_{m} \cdot n \lambda_{M}}{\mathrm{~A}(s)(s+a)} F_{\Phi_{1}}\left(s+\lambda_{m}^{\prime}+n \lambda_{M}+\delta\right) \cdot F_{\Phi_{1}}(s) \\
& \overline{\mathrm{P}}_{6}(s)=\frac{n \lambda_{M}}{\mathrm{~A}(s)(s+a)} \\
& \overline{\mathrm{P}}_{7}(s)=\frac{a \cdot n \lambda_{M}}{\mathrm{~A}(s)(s+a)} F_{\Phi_{1}}(s) \\
& \overline{\mathrm{P}}_{8}(s)=\frac{\delta}{(s+\mu)} \frac{1}{\mathrm{~A}(\mathrm{~s})}\left\{1+2 \lambda_{m} \cdot F_{\Phi_{1}}\left(s+\lambda_{m}^{\prime}+n \lambda_{M}+\delta\right)\right\}
\end{aligned}
$$

When all repairs follow the exponential time distribution

Setting $\bar{S}_{\Phi_{i}}=\frac{\Phi_{i}}{s+\Phi_{i}}, i=1,2$, we get

$$
\begin{aligned}
& \overline{\mathrm{P}}_{0}(s)=\frac{1}{\mathrm{~B}(s)} \text {, where } \\
& \mathrm{B}(s)=2 \lambda_{m}+n \lambda_{M}+\delta-2 \lambda_{m} \cdot \frac{\Phi_{1}}{\left(s+\lambda^{\prime}{ }_{m}+n \lambda_{M}+\delta+\Phi_{1}\right)} \\
& -\frac{2 a \lambda_{m}^{\prime} \lambda_{m}}{(s+a)} \frac{\Phi_{1}}{\left(s+\lambda_{m}^{\prime}+n \lambda_{M}+\delta+\Phi_{1}\right)} \frac{\Phi_{2}}{\left(s+\Phi_{2}\right)} \\
& -2 a \lambda_{m} \lambda_{m}^{\prime} \cdot \frac{\Phi_{1}}{\left(s+\lambda_{m}^{\prime}+n \lambda_{M}+\Phi_{1}+\delta\right)} \frac{\Phi_{2}}{\left(s+\Phi_{2}\right)}-\frac{n \lambda_{M} \cdot a}{(s+a)} \frac{\Phi_{1}}{\left(s+\Phi_{1}\right)} \\
& +\frac{\delta}{(s+\mu)}\left\{1+2 \lambda_{m} \cdot \frac{\Phi_{1}}{\left(s+\lambda_{m}^{\prime}+n \lambda_{M}+\delta+\Phi_{1}\right)}\right\} \\
& \overline{\mathrm{P}}_{1}(s)=\frac{2 \lambda_{m}}{\mathrm{~B}(s)} \frac{1}{\left(s+\lambda_{m}^{\prime}+n \lambda_{M}+\Phi_{1}+\delta\right)} \\
& \overline{\mathrm{P}}_{2}(s)=\frac{2 \lambda_{m}^{\prime} \lambda_{m}}{\mathrm{~B}(s)(s+a)} \frac{1}{\left(s+\lambda_{m}^{\prime}+n \lambda_{M}+\Phi_{1}+\delta\right)} \\
& \overline{\mathrm{P}}_{3}(s)=\frac{2 a \lambda_{m}^{\prime} \lambda_{m}}{\mathrm{~B}(s)(s+a)} \frac{1}{\left(s+\lambda_{m}^{\prime}+n \lambda_{M}+\Phi_{1}+\delta\right)} \frac{1}{\left(s+\Phi_{2}\right)} \\
& \overline{\mathrm{P}}_{4}(s)=\frac{2 a \lambda_{m} \cdot n \lambda_{M}}{\mathrm{~B}(s) \cdot(s+a)} \frac{1}{\left(s+\lambda_{m}^{\prime}+n \lambda_{M}+\Phi_{1}+\delta\right)}
\end{aligned}
$$




$$
\begin{aligned}
& \overline{\mathrm{P}}_{5}(s)=\frac{2 a \lambda_{m} \cdot n \lambda_{M}}{\mathrm{~B}(s)(s+a)} \frac{1}{\left(s+\lambda_{m}^{\prime}+n \lambda_{M}+\delta\right)} \frac{1}{\left(s+\Phi_{2}\right)} \\
& \overline{\mathrm{P}}_{6}(s)=\frac{n \lambda_{M}}{\mathrm{~B}(s) \cdot(s+a)} \\
& \overline{\mathrm{P}}_{7}(s)=\frac{a \cdot n \lambda_{M}}{\mathrm{~B}(s)(s+a)} \frac{1}{\left(\mathrm{~s}+\Phi_{1}\right)} \\
& \overline{\mathrm{P}}_{8}(s)=\frac{\delta}{\mathrm{B}(s)(s+\mu)}\left\{1+\frac{2 \lambda_{m}}{\left(s+\lambda_{m}+n \lambda_{M}+\Phi_{1}+\delta\right)}\right\}
\end{aligned}
$$

It can be easily verified that

$$
\sum_{i=1}^{8} P_{i}(s)=\frac{1}{s}
$$

Evaluation of Up State Probability

$$
\begin{aligned}
& \overline{\mathrm{P}}_{u p}(s)=\overline{\mathrm{P}}_{0}(s)+\overline{\mathrm{P}_{1}}(s) \\
& \overline{\mathrm{P}}_{u p}(s)=\frac{1}{\left(s+2 \lambda_{m}+n \lambda_{M}+\delta\right)}\left[1+\frac{2 \lambda_{m}}{\left(s+\lambda_{m}^{\prime}+n \lambda_{M}+\delta\right)}\right], \text { by inversion process } \\
& \mathrm{P}_{u p}(t)=\frac{1}{\left(2 \lambda_{m}-\lambda_{m}^{\prime}\right)}\left[2 \lambda_{m} \cdot \exp \left\{-\left(\left(\lambda_{m}^{\prime}+n \lambda_{M}+\delta\right) t\right)\right\}-\lambda_{m}^{\prime} \cdot \exp \left\{-\left(2 \lambda_{m}+\lambda_{m}^{\prime}+\delta\right) t\right\}\right]
\end{aligned}
$$

To obtain expression for reliability, setting all repair rates zero in equation (33). We get

$$
\begin{aligned}
& \bar{R}(s)=\frac{1}{\left(s+2 \lambda_{m}+n \lambda_{M}+\delta\right)}, \text { by inversion process we get } \\
& R(t)=\exp \left\{-\left(2 \lambda_{m}+n \lambda_{M}+\delta\right) t\right\} \\
& H(t)=\frac{C_{1}}{\left(2 \lambda_{m}-\lambda_{m}^{\prime}\right)}\left[\frac{2 \lambda_{m}\left(1-\exp \left\{-\left(\lambda_{m}^{\prime}+n \lambda_{M}+\delta\right)\right\}\right)}{\left(\lambda_{m}^{\prime}+n \lambda_{M}+\delta\right)}-\lambda^{\prime} m\left(\frac{1-\exp \left\{-\left(2 \lambda_{m}+\lambda^{\prime} m+\delta\right)\right\}}{\left(2 \lambda_{m}+\lambda_{m}^{\prime}+\delta\right)}\right)\right]-C_{2} t
\end{aligned}
$$

\section{V.T.T.F of the System}

$$
\sigma^{2}=-2 \lim _{s \rightarrow 0} \frac{d R(s)}{d s}-(\text { M.T.S.F })^{2} \Rightarrow \sigma^{2}=\frac{2}{\left\{2 \lambda_{m}+n \lambda_{M}+\delta\right\}^{2}}-\frac{1}{\left(2 \lambda_{m}+n \lambda_{M}+\delta\right)^{2}}
$$

\section{Mean Time to System Failure}

$$
\text { M.T.S.F }={ }_{S \rightarrow 0}^{\operatorname{Lim}} R(s) \Rightarrow \text { M.T.S.F }=\frac{1}{2 \lambda_{m}+n \lambda_{M}+\delta}
$$


Numerical computations

Reliability Analysis: Taking and varying t from 0 to 20,in equation (35),we obtain

Table 1.0

\begin{tabular}{|c|c|c|c|}
\hline $\mathbf{T}$ & $\mathbf{R}(\mathbf{t})$ for $\mathbf{n}=\mathbf{1}$ & $\mathbf{R}(\mathbf{t}) \mathbf{f o r} \mathbf{n}=\mathbf{2}$ & $\mathbf{R}(\mathbf{t}) \mathbf{f o r} \mathbf{n}=\mathbf{j}$ \\
\hline 0 & 1 & 1 & 1 \\
\hline 1 & .9598291299 & .9408232398 & 0.922193691 \\
\hline 2 & .9212719587 & .8851436850 & .8504412045 \\
\hline 3 & .8842636626 & .8327681557 & .7842715138 \\
\hline 4 & .8487420219 & .7834876343 & .7232502424 \\
\hline 5 & .8146473164 & .7371233744 & .6669768109 \\
\hline 6 & .7819222490 & .6935028012 & .6150818073 \\
\hline 7 & .7505117288 & .6524635522 & .5672245624 \\
\hline 8 & .7203630197 & .6138528730 & .5230909131 \\
\hline 9 & .6914254105 & .5775270488 & .4823911401 \\
\hline 10 & .6636502500 & .5433508691 & .4448580662 \\
\hline 11 & .6369908422 & .5111971250 & .4102453023 \\
\hline 12 & .6114023658 & .4809461353 & .3783256297 \\
\hline 13 & .5868418008 & .4524853012 & .3488950900 \\
\hline 14 & .5632678551 & .4257086870 & .3217437042 \\
\hline 15 & .5406408953 & .4005166261 & .2967100143 \\
\hline 16 & .5189228802 & .3768153497 & .2736241034 \\
\hline 17 & .4980690982 & .3545166381 & .2523344220 \\
\hline 18 & .4706909820 & .3335374921 & .2327012121 \\
\hline 19 & .4588646466 & .3137998239 & .2145955898 \\
\hline 20 & .4404336545 & .2952301669 & .1978986991 \\
\hline
\end{tabular}

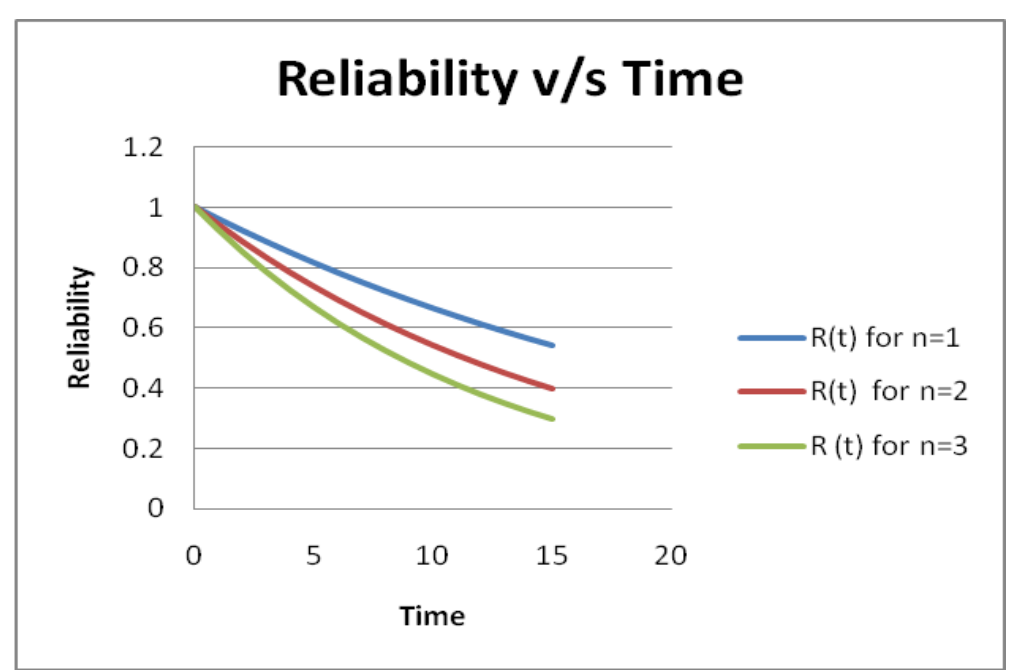

Figure 1.0 


\section{Cost Function Analysis}

Taking $\lambda_{m}=0.001, \lambda_{m}^{\prime}=0.003, \lambda_{M}=0.002, \delta=0.001, C_{1}=100$, for $C_{2}=50,60,70$, in equation (36), we get

(a) For, $n=1$

Table 1.2 (a)

\begin{tabular}{|c|c|c|c|}
\hline $\boldsymbol{T}$ & $\boldsymbol{H}(\boldsymbol{t}) \boldsymbol{C}_{\mathbf{2}}=\mathbf{5 0}$ & $\boldsymbol{H}(\boldsymbol{t}) \boldsymbol{C}_{\mathbf{2}}=\mathbf{6 0}$ & $\boldsymbol{H}(\boldsymbol{t}) \boldsymbol{C}_{\mathbf{2}}=\mathbf{7 0}$ \\
\hline 0 & 0 & 0 & 0 \\
\hline 1 & 48.46589330 & 38.46589330 & 28.46589330 \\
\hline 2 & 93.92617164 & 73.92617167 & 53.92617167 \\
\hline 3 & 136.4725797 & 106.4725797 & 76.47257970 \\
\hline 4 & 176.1940617 & 136.1940616 & 96.19406170 \\
\hline 5 & 213.1768468 & 163.1768468 & 113.1768468 \\
\hline 6 & 247.5045323 & 187.5045323 & 127.5045323 \\
\hline 7 & 279.2581635 & 209.2581635 & 149.2581635 \\
\hline 8 & 308.5163120 & 228.5163120 & 148.5163120 \\
\hline 9 & 335.3551509 & 245.3551509 & 155.3551509 \\
\hline 10 & 359.8485283 & 259.8485283 & 159.8485283 \\
\hline
\end{tabular}

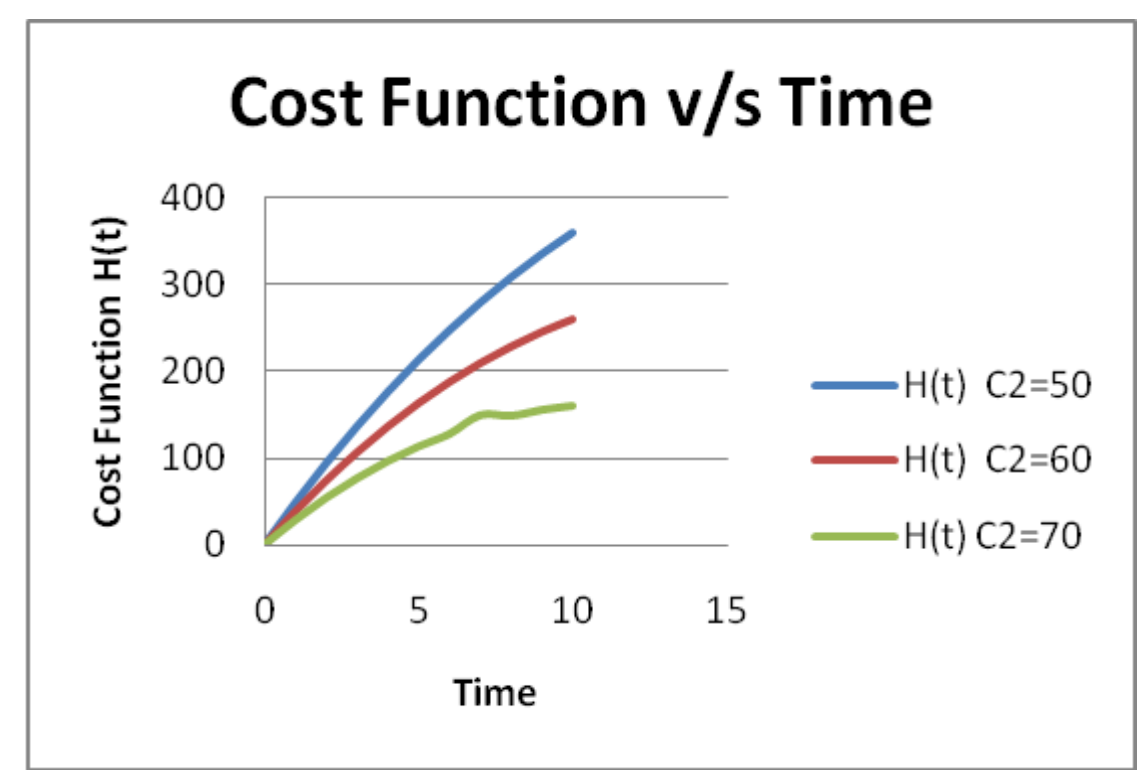

Figure 1.2 (a) 
(b) For, $n=2$

Table 1.2 (b)

\begin{tabular}{|c|c|c|c|}
\hline $\mathbf{T}$ & $\mathbf{H}(\mathbf{t}), \mathbf{C}_{2}=\mathbf{5 0}$ & $\mathbf{H}(\mathbf{t}), \mathbf{C}_{2}=\mathbf{6 0}$ & $\mathbf{H}(\mathbf{t}), \mathbf{C}_{2}=\mathbf{7 0}$ \\
\hline 0 & 0 & 0 & 0 \\
\hline 1 & 46.51971250 & 36.51971245 & 26.51971245 \\
\hline 2 & 86.35009632 & 66.35009632 & 46.35009632 \\
\hline 3 & 119.8814524 & 89.88145242 & 59.88145242 \\
\hline 4 & 147.4829141 & 107.4829141 & 67.48291410 \\
\hline 5 & 169.5035533 & 119.5035533 & 69.50355333 \\
\hline 6 & 186.2734306 & 126.2734306 & 58.10459095 \\
\hline 7 & 198.1045904 & 128.1045904 & 45.29200634 \\
\hline 8 & 205.1144769 & 118.1147690 & 58.10459045 \\
\hline 9 & 208.1144769 & 106.8354761 & 28.11447640 \\
\hline
\end{tabular}

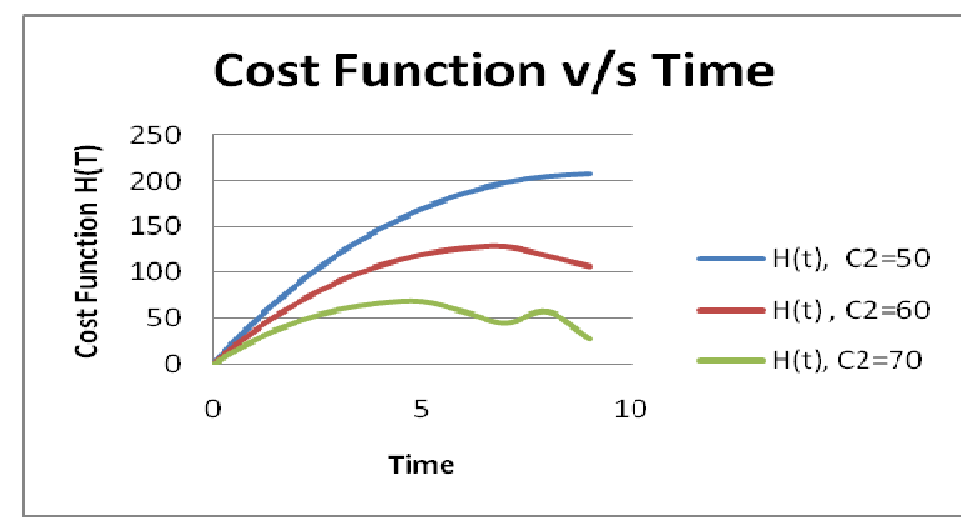

Figure 1.2 (b)

(c) For, $n=3$

Table 1.2 (c)

\begin{tabular}{|c|c|c|c|}
\hline $\mathbf{T}$ & $\mathbf{H . 1}(\mathbf{t}), \mathbf{C}_{2}=\mathbf{5 0}$ & $\mathbf{H}(\mathbf{t}), \mathbf{C}_{\mathbf{2}}=\mathbf{6 0}$ & $\mathbf{H}(\mathbf{t}), \mathbf{C}_{2}=\mathbf{7 0}$ \\
\hline 0 & 0 & 0 & 0 \\
\hline 1 & 44.59919930 & 34.59919930 & 24.59919930 \\
\hline 2 & 78.97169741 & 58.97169741 & 38.97169741 \\
\hline 3 & 103.9326676 & 73.93266759 & 43.93266675 \\
\hline 4 & 120.2379251 & 80.23792507 & 54.23979250 \\
\hline 5 & 128.5881019 & 78.58810188 & 60.58810188 \\
\hline 6 & 129.6325321 & 69.63253213 & 48.63253213 \\
\hline 7 & 123.9728680 & 53.97286799 & 40.97286799 \\
\hline 8 & 112.1664470 & 32.16644766 & 34.14644766 \\
\hline 9 & 94.72941189 & 28.72929411 & 30.72929411 \\
\hline 10 & 72.13964768 & 20.13964768 & 19.13964768 \\
\hline
\end{tabular}




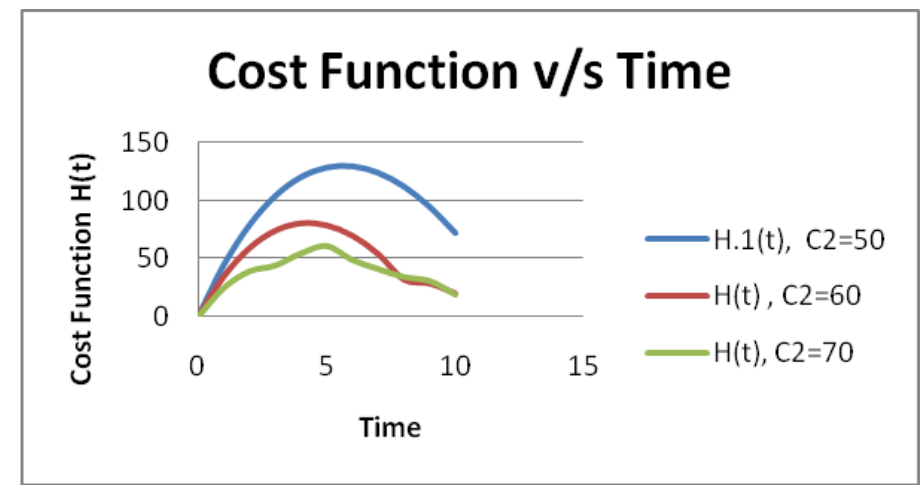

Figure 1.2 (c)

Variance of time to failure: Taking $\lambda_{m}=0.001, \delta=0.001, \lambda_{M}=0.002$ in equation (37) for $n=1,2,3$ we get

Table 1.30

\begin{tabular}{|c|c|c|c|}
\hline \multirow{2}{*}{ S.No } & \multicolumn{3}{|c|}{ Variance of time to failure } \\
\cline { 2 - 4 } & $\mathrm{n}=1$ & $\mathrm{n}=2$ & $\mathrm{n}=3$ \\
\hline 1 & 31.2500 & 62.5000 & 75.555550 \\
\hline 2 & 23.8095 & 38.4615 & 45.714285 \\
\hline 3 & 19.2308 & 27.7778 & 34.239032 \\
\hline 4 & 16.1290 & 21.7391 & 29.502555 \\
\hline 5 & 13.8889 & 17.8571 & 24.675182 \\
\hline 6 & 12.1951 & 15.1515 & 18.023752 \\
\hline 7 & 10.8696 & 13.1579 & 16.765832 \\
\hline 8 & 9.80392 & 11.6279 & 14.675182 \\
\hline 9 & 8.92857 & 10.4167 & 12.062875 \\
\hline 10 & 9.61538 & 9.43396 & 9.353827 \\
\hline
\end{tabular}

Variance of Time to Failure v/s S.No.

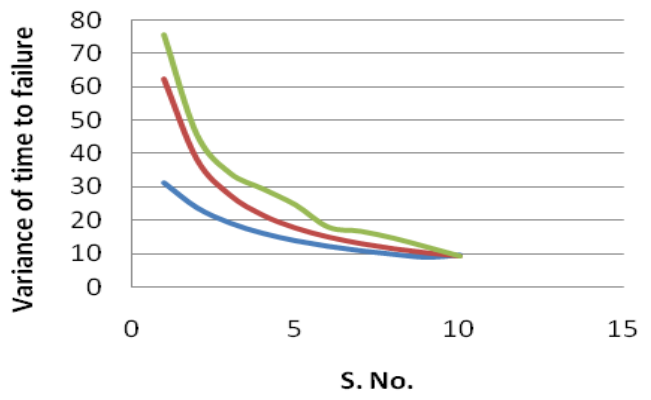




\section{Conclusion of This paper / Result}

- From the Table 1.0 and Graph 1.0 authors conclude that reliability of this P-S system decreases as well as time increase i.e. as time t increase reliability of the system decreases.

- $\quad$ By the inspection, Table 1.2 (a), 1.2(b) \& 1.2(c) and graph fig 1.2(a), fig1.2 (b),\& fig-1.2(c) .Expected profit $\mathrm{H}(\mathrm{t})$ decrease rapidly as $\mathrm{C} 2$ increase and there is no harm by using n identical unit except initial burden.

- $\quad$ By the Inspection Table 1.30\& graph , fig 1.30 if P-S system consist by more than two identical unit than variance of time to failure is large but for less than two units it is small, while for a long internal it being constant.

\section{References}

[1] Kazu okumoto; Availability of 2-component dependent system. IEEE TransReliability. R-30(2), 205205(1981).

[2] Dhillon B.S; Partial and Catastrophic failure analysis of a complex system" Microelectronics Reliability. Vol.19 (4), 349-352(1979).

[3] Murty A.S.R; Verma A.K; "Reliability Modeling of Electronic Components" Microelectronics Reliability Vol.-27(1), pp. 29-32(1987)

[4] Panday D; Jacob Mendus; "Cost analysis, Availability and M.T.T.F of a 3 state stand by complex system under common cause and human failure" Microelectronic reliability 35(1), 91-95(1995)

[5] Gupta, Rakesh; Choudhary, Alka; "Profit Analysis of a Two-Unit ManMachine system with random appearance and disappearance of the operator". Microelectronics Reliability. 34(6), 1133-1136 (1994).

[6] Kumar Sachin; Tyagi Anand; "Evaluation of Some Reliability Parameters of a Three State Repairable System with Environmental Failure, published in International Journal of Research and Review in Applied Sciences.Vol.1, Issue-2, pp. 96-103, 2009.

[7] Kumar Sachin; Tyagi Anand; "Operational Study of a Standby System Incorporating the Concept of Delay in Time with Critical Human Error and Environmental Failure”. International Journal of Computational Science and Mathematics, Vol. 3, No. 1, pp. 127-132, 2011 
